\title{
Laparoscopic and Open Colorectal Surgery in Previously Operated Patients is Associated with Considerable Need for Adhesiolysis
}

\author{
Jyrki Kössi $^{*}, 1$, Markku Luostarinen ${ }^{1}$ and Matti Laato ${ }^{2}$ \\ ${ }^{I}$ Department of Surgery, Päijät-Häme Central Hospital, Keskussairaalankatu 7, 15850 Lahti, Finland \\ ${ }^{2}$ Department of Surgery, University of Turku, Kiinamyllynkatu 4-8, 20520 Turku, Finland
}

\begin{abstract}
Background: Adhesions caused by previous operations increase operative time and the risk of peroperative complications as well as conversions. The aim of this study was to evaluate the complications and the extra operative time related to the dividing of adhesions caused by previous surgery in patients scheduled for elective colorectal surgery. Methods: In a consecutive series of patients with previous abdominal or pelvic surgery and scheduled for open or laparoscopic colorectal procedures, data on patient demography, previous operations, index operations, intraoperative complications, conversions, adhesion division time and the extra time needed for first trocar insertion were collected prospectively. Results: Data from 111 patients were collected. There were 29 open and 80 laparoscopic operations, and conversion was needed in two patients due to adhesions. The mean extra time needed to divide adhesions was 19.9 min (range 0.2-120 $\mathrm{min}$ ) in open operations, $35.4 \mathrm{~min}$ (range 22.9-48 $\mathrm{min}$ ) in converted cases and 9.5 $\mathrm{min}$ (range 0-67 $\mathrm{min}$ ) in laparoscopic operations. The extra time corresponded for $12 \%$ of total operative time. The mean extra time needed to insert the first trocar was $1 \mathrm{~min}$ (range 0-8 min). There were two serosal lesions necessitating suturation (one in open and one in laparoscopic operation) and one inadvertent enterotomy. The extra time needed to divide adhesions was correlated with the number of previous operations. Conclusions: Adhesions caused by previous surgery increase operative time considerably. The increase correlates with the number of previous operations. The adhesiolysis is associated with certain amount of intraoperative complications.
\end{abstract}

Keywords: Adhesions, conversion rate, complications, previous surgery, surgery duration, surgical workload.

\section{INTRODUCTION}

The almost inevitable formation of postoperative adhesions after laparotomy is a well-recognized phenomenon, and the epidemiology, workload and cost of postoperative adhesion-related problems have been well documented in recent studies [1-4]. According to Ellis and coworkers [1], roughly one-third of patients having had open abdominal surgery are readmitted due to a disorder possibly related to adhesions during the subsequent ten years, and over half of their readmissions are due to repeat surgery that is potentially complicated by the adhesions. However, only a limited number of reports have been published that clarify the magnitude of the difficulties encountered when re-entering the abdomen. These publications have mainly focused on the time needed to divide previous adhesions or on intraoperative complications caused by them, and the studies have included only open operations [5-7]. Therefore, more information concerning the issue is urgently needed, especially in view of the lack of detailed data regarding adhesion-related problems in laparoscopic operations. The primary aim of this study was to evaluate the time needed to divide postoperative adhesions and secondarily to record the intraoperative complications related to adhesions in general surgical practice including both open and laparoscopic colorectal procedures.

*Address correspondence to this author at the Department of Surgery, Päijät-Häme Central Hospital, Keskussairaalankatu 7, 15850 Lahti, Finland; Tel: +358-44-7195965; Fax: +358-3-8192710;

E-mail: jyrki.kossi@phsotey.fi

\section{PATIENTS AND METHODS}

A consecutive series of 111 patients scheduled for elective colorectal surgery and having prior abdominal or pelvic surgery were included in the study. All of the operations were performed by one of the authors (JK). Information on patient demography, previous surgery, intraoperative complications and the time taken to divide relevant adhesions during the operation was collected prospectively. In laparoscopic operations, the extra time needed to fill the abdominal cavity with carbon dioxide and to place the first trocar was measured as well. The Veress needle technique was used routinely in our practice, and the open technique to place a trocar and to fill abdominal cavity was used only when necessary. However, the Veress needle was inserted into the abdomen from a site that was judged to pose the lowest risk for adhesions. Adhesions were regarded as relevant if it was necessary to divide them in order to complete the scheduled operation, to place the abdominal retractor properly or to place trocars properly in laparoscopic operations. Thus, adhesions remote from the surgical site and nonrelevant small bowel adhesions were not divided. The time needed to divide adhesions was measured by stopwatch. Special attention was paid to inflammatory adhesions (e.g. adhesions related to diverticulitis) in order to exclude them from the recorded adhesion division time. The study was approved by the local ethics committee. Statistical analyses were performed using SPSS software (SPSS for Windows, version 15.0, SPSS, Chicago, Il, USA). For categorical variables, a chi-square test was used, and for continuous 
variables two-way ANOVA was used. A p value lower than 0.05 was considered statistically significant.

\section{RESULTS}

The study group comprised 111 patients of which 25 were men and 86 women. The mean age of the patients was 60.8 years (range 25-84.75), and they had altogether 189 previous abdominal or pelvic operations. Sixty-four patients (57.7\%) had one previous operation, 23 patients $(20.7 \%)$ two, 17 patients $(15.3 \%)$ three, five patients $(4.5 \%)$ four and two patients $(1.8 \%)$ more than four previous operations. Only $12(6.3 \%)$ of the previous operations had been performed laparoscopically. The most common previous operations were as follows: 36 appendectomies (17.5\%), 34 hysterectomies $(18.0 \%), 12$ hysterectomies in combination with another gynecological procedure $(6.3 \%), 18$ open cholecystectomies (9.5\%), and 23 colorectal operations (12.2\%). These operations constituted roughly two-thirds of the previous operations. The type and number of performed index operations are shown in Table 1 . Twenty-nine $(26.1 \%)$ of the operations were intentionally performed openly, and 80 (72.1\%) were completed laparoscopically. There were only two conversions due to adhesions $(2.5 \%$ of the laparoscopic operations).

Table 1. The Type and Number of Operations During which the Adhesion Division Time was Measured

\begin{tabular}{|c|c|c|}
\hline \multicolumn{2}{|c|}{ Type of Operation } & Number of Operation \\
\hline \multicolumn{2}{|c|}{ Right hemicolectomy } & 17 \\
\hline \multirow{2}{*}{$\begin{array}{l}\text { Laparoscopic } \\
\text { Open }\end{array}$} & 9 & \\
\hline & 8 & \\
\hline \multicolumn{2}{|c|}{ Sigmoid resection } & 52 \\
\hline \multirow{2}{*}{$\begin{array}{l}\text { Laparoscopic } \\
\text { Open }\end{array}$} & 48 & \\
\hline & 4 & \\
\hline \multicolumn{2}{|c|}{ Rectal resection } & 19 \\
\hline \multirow{2}{*}{$\begin{array}{l}\text { Laparoscopic } \\
\text { Open }\end{array}$} & 11 & \\
\hline & 8 & \\
\hline \multicolumn{2}{|c|}{ Hartmann reversal } & 6 \\
\hline \multirow[t]{2}{*}{ Open } & 6 & \\
\hline & & 9 \\
\hline \multirow[t]{2}{*}{ Laparoscopic } & 9 & \\
\hline & & 8 \\
\hline \multirow{2}{*}{$\begin{array}{l}\text { Laparoscopic } \\
\text { Open }\end{array}$} & 4 & \\
\hline & 4 & \\
\hline
\end{tabular}

The mean total adhesion division time represented roughly $12 \%$ of the mean operative time. The mean and median time needed to divide postoperative adhesions, as well as extra time needed to position the first trocar, are shown in Table $\mathbf{2}$. In our series it took roughly twice as long to divide adhesions in open procedures, and this difference was statistically significant $(\mathrm{p}=0.003)$. However, extensive previous procedures (such as colorectal operations) were more common in the open group $(p<0.001)$, although the distribution of the number of previous operations was comparable between groups. In the majority of patients $(60.4 \%)$, it took less than 10 minutes to divide adhesions (Fig. 1). As expected, the mean adhesion division time was longer in patients who had more previous operations (Table 3).

Table 2. Mean and Median Adhesion Division Time (min) and the Extra Time Needed to Insert the First Trocar in Laparoscopic Operations. Adhesion Division Time, $p=0.003$, ANOVA

\begin{tabular}{|l|c|c|c|}
\hline & Mean & Median & Range \\
\hline \hline Open & 19.9 & 11.0 & $0.2-120$ \\
\hline Converted & 35.4 & 35.4 & $22.9-48$ \\
\hline Laparoscopic & & & \\
\hline$\quad$ Adhesion division & 9.5 & 4.0 & $0-67$ \\
\hline$\quad$ Trocar insertion & 1.0 & 0 & $0-8$ \\
\hline Total (adhesion division) & 12.6 & 6.0 & $0-120$ \\
\hline
\end{tabular}

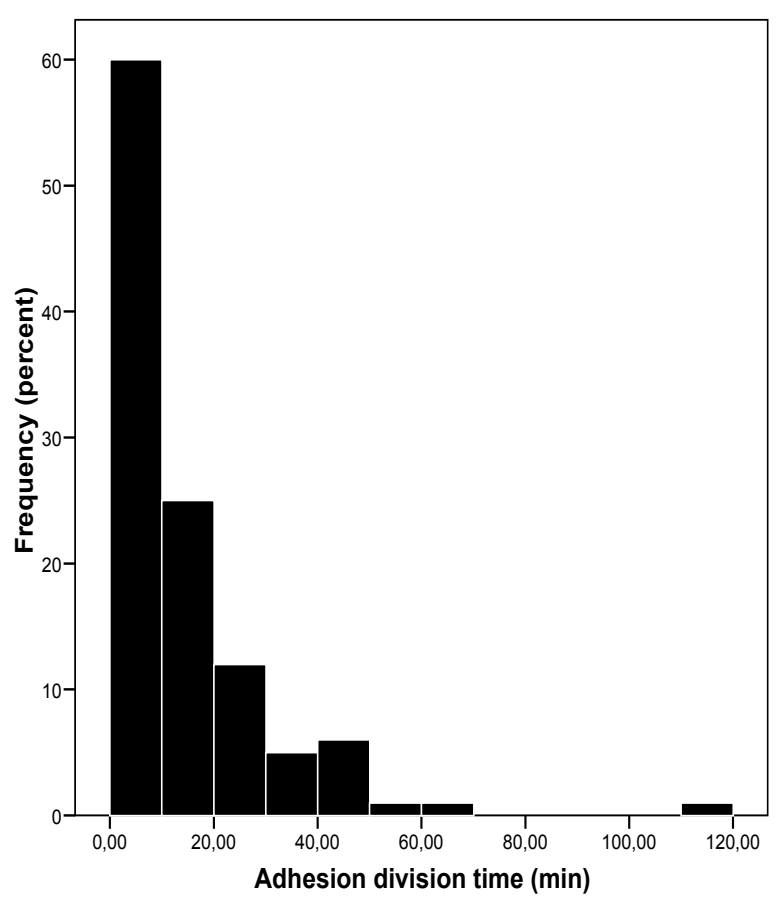

Fig. (1). Distribution (percent) of adhesion division time among patients.

There was only one $(0.9 \%)$ inadvertent enterotomy and two serosal lesions $(1.8 \%$, one in open operation, one in laparoscopic) that required suturation. The enterotomy occurred in a laparoscopic operation. No other intraoperative complications occurred due to adhesiolysis. 
Table 3. The Comparison of Mean Adhesion Division Time in Patients having a Different Number of Previous Operations. $p=0.007$, ANOVA

\begin{tabular}{|c|c|c|}
\hline $\begin{array}{c}\text { Number of previous } \\
\text { operations }\end{array}$ & $\begin{array}{c}\text { Mean adhesion } \\
\text { division time }\end{array}$ & Range \\
\hline \hline 1 & 9.0 & $0-57$ \\
\hline 2 & 11.2 & $0-67$ \\
\hline 3 & 25.6 & $0-120$ \\
\hline 4 & 21.4 & $6-42$ \\
\hline 5 or more & 14.4 & $6-22.9$ \\
\hline
\end{tabular}

\section{DISCUSSION}

Ellis and coworkers [1] have estimated that the rate of reoperations potentially complicated by adhesions per 100 initial abdominal procedures is roughly 40 during a 10 -year follow-up. In spite of this important clinical finding, few studies have focused on the difficulties encountered when reentering the abdomen. These studies have evaluated the extra incision or opening time, adhesion division time and the frequency of inadvertent small bowel enterotomy [5-7]. However, all of these studies have limitations since they included emergency operations and only open procedures. Furthermore, the type of previous or index operations was not always defined exactly. The current trend in general and colorectal surgery is towards laparoscopic operations, which have specific features concerning previous adhesions: the establishment of a pneumoperitoneum, insertion of the first trocar, a presumed increase in the frequency of intraoperative enterotomies and an increased risk for conversion due to previous adhesions. Although some studies have evaluated the effect of previous surgery on laparoscopic surgery in general [8-10], there are no detailed data available regarding the adhesion division time in laparoscopic surgery. Our study brings out important information in order to fill this gap.

The number of conversions $(2.5 \%)$ and intraoperative adhesion-related complications $(2.7 \%)$ in our series was considerably low. Conversion to open surgery was necessary in $16.5-26.1 \%$ of patients in previous reports [9, 11-13]. In addition, the incidence of intraoperative bowel injury associated with adhesiolysis varies from 0 to $17.6 \%[13,14]$ in laparoscopic operations and may be as high as $19 \%$ in open surgery [7]. The reason for the low incidence of bowel injury in our series is not evident, but there are at least two possible explanations. Firstly, it is very likely that the patient profile in our series is different and includes more "easy" cases with more minor types of previous operations. Indeed, a great proportion of the patients in the laparoscopic group had only an appendectomy in their history. Secondly, the prospective setting of the study may have had an impact on the results. It may be the case that surgeons perform operations more cautiously in prospective studies compared to the average practice. However, it can be concluded that, in general surgical laparoscopic practice performed by an experienced surgeon, the incidence of serious complications associated with the division of previous adhesions is low, justifying the laparoscopic approach.
The mean (or median) adhesion division time, including extra incision time, has been 18-33.5 min in previous studies involving open operations $[5,6]$. In a case-control study including laparoscopic colorectal operations, the operative time was 26 min longer in patients with previous surgery [13]. Our open surgery results are well in line with these previous reports $(21 \mathrm{~min}$ in open surgery and $35 \mathrm{~min}$ in converted operations). However, the adhesion division time in our laparoscopic group is shorter compared to the aforementioned previous study (10.5 min, which includes the extra time needed for the first trocar insertion). The reason for this discrepancy is quite evidently that Vignali and coworkers [13] excluded patients with only an appendectomy in their past. Surprisingly, the adhesion division time in our study was significantly shorter in laparoscopic operations. This finding is, however, heavily biased by the difference of patient material between the open and laparoscopic groups. The patients in the open group had more extensive operations in their history. It is worth mentioning that, although some patients require extensive adhesiolysis, adhesions in the majority of patients can be divided within ten minutes, according to our data.

In conclusion, the findings of our study confirm that previous surgery increases operative time considerably in both open and laparoscopic colorectal surgery, and this increase correlates with the number of previous operations. Further, it seems that laparoscopic colorectal surgery in previously operated patients is safe.

\section{DISCLAIMER}

The results have been presented in part at the VIII PAX meeting on September 18 to 20, 2008, Clermont-Ferrand, France.

\section{REFERENCES}

[1] Ellis H, Moran BJ, Thompson JN, et al. Adhesion-related hospital readmissions after abdominal and pelvic surgery: a retrospective cohort study. Lancet 1999; 353: 1476-80.

[2] Beck DE, Opelka FG, Bailey HR, et al. Incidence of small-bowel obstruction and adhesiolysis after open colorectal and general surgery. Dis Colon Rectum 1999; 42: 241-8.

[3] Menzies D, Parker M, Hoare R, et al. Small bowel obstruction due to postoperative adhesions: treatment patterns and associated costs in 110 hospital admissions. Ann R Coll Surg Engl 2001; 83: 40-6.

[4] Kössi J, Salminen P, Rantala A, et al. Population-based study of the surgical workload and economic impact of bowel obstruction caused by postoperative adhesions. Br J Surg 2003; 90: 1441-4.

[5] Beck DE, Ferguson MA, Opelka FG, et al. Effect of previous surgery on abdominal opening time. Dis Colon Rectum 2000; 43: 1749-53.

[6] Coleman MG, McLain AD, Moran BJ. Impact of previous surgery on time taken for incision and division of adhesions during laparotomy. Dis Colon Rectum 2000; 43: 1297-9.

[7] van der Krabben AA, Dijkstra FR, Nieuwenhuijzen M, et al. Morbidity and mortality of inadvertent enterotomy during adhesiotomy. Br J Surg 2000; 87: 467-71.

[8] Karayiannakis AJ, Polychronidis A, Perente S, et al. Laparoscopic cholecystectomy in patients with previous upper or lower abdominal surgery. Surg Endosc 2004; 18: 97-101.

[9] Law WL, Lee YM, Chu KW. Previous abdominal operations do not affect the outcomes of laparoscopic colorectal surgery. Surg Endosc 2005; 19: 326-30.

[10] Wu J-M, Lin H-F, Chen K-H, et al. Impact of previous abdominal surgery on laparoscopic appendectomy for acute appendicitis. Surg Endosc 2007; 21: 570-3. 
[11] Hamel CT, Pikarsky E, Weiss E, et al. Do prior abdominal surgery alter the outcome of laparoscopically assisted right hemicolectomy? Surg Endosc 2000; 14: 853-7.

[12] Gonzales IA, Malagon AM, Lopex-Tomassetti F, et al. Impact of previous abdominal surgery on colorectal laparoscopy results: a comparative clinical study. Surg Laparosc Percutan Tech 2006; 16: $8-11$.
[13] Vignali A, Di Palo S, De Nardi P, et al. Impact of previous abdominal surgery on the outcome of laparoscopic colectomy: a case control study. Tech Coloproctol 2007; 11: 241-6.

[14] Szomstein S, Lo Menzo E, Simplendorfer C, et al. Laparoscopic lysis of adhesions. World J Surg 2006; 30: 535-40.

Received: July 01, 2010

(C) Kössi et al.; Licensee Bentham Open.

This is an open access article licensed under the terms of the Creative Commons Attribution Non-Commercial License (http://creativecommons.org/licenses/by$\mathrm{nc} / 3.0 /$ ), which permits unrestricted, non-commercial use, distribution and reproduction in any medium, provided the work is properly cited. 